\title{
Detection of Methoxymethanol as a Photochemistry Product of Condensed Methanol
}

\author{
Hope Schneider, ${ }^{1}$ Anna Caldwell-Overdier, ${ }^{1}$ Sophie Coppieters ' $t$ Wallant, ${ }^{1}$ Lan Dau, ${ }^{1}$ Jean
} Huang, ${ }^{1}$ Ifunanya Nwolah, ${ }^{1}$ Muhammad Kasule, ${ }^{2}$ Christina Buffo, ${ }^{1}$ Ella Mullikin, ${ }^{1}$ Lily Widdup, ${ }^{1}$ Aury Hay, ${ }^{1}$ Si Tong Bao, ${ }^{1}$ Jeniffer Perea, ${ }^{1}$ Mayla Thompson, ${ }^{1}$ Rhoda Tano-Menka, ${ }^{1}$ Mileva Van Tuyl, ${ }^{1}$ Amy Wang, ${ }^{1}$ Sophia Bussey, ${ }^{1}$ Nina Sachdev, ${ }^{1}$ Christine Zhang, ${ }^{1}$ Michael C. Boyer, ${ }^{2}$ and Christopher R. Arumainayagam ${ }^{1 *}$

${ }^{1}$ Department of Chemistry, Wellesley College, Wellesley, MA 02481

${ }^{2}$ Department of Physics, Clark University, Worcester, MA 01610

\begin{abstract}
We report the identification of methoxymethanol $\left(\mathrm{CH}_{3} \mathrm{OCH}_{2} \mathrm{OH}\right)$ as a photochemistry product of condensed methanol $\left(\mathrm{CH}_{3} \mathrm{OH}\right)$ based on temperature-programmed desorption studies conducted following photon irradiation at energies below the ionization threshold $(9.8 \mathrm{eV})$ of condensed methanol. The first detection of methoxymethanol in the interstellar medium was reported in 2017 based on data from Bands 6 and 7 from the Atacama Large Millimeter/submillimeter Array (ALMA). The cosmic synthesis of "complex" organic molecules such as methyl formate $\left(\mathrm{HCOOCH}_{3}\right)$, dimethyl ether $\left(\mathrm{CH}_{3} \mathrm{OCH}_{3}\right)$, acetic acid $\left(\mathrm{CH}_{3} \mathrm{COOH}\right)$, ethylene glycol $\left(\mathrm{HOCH}_{2} \mathrm{CH}_{2} \mathrm{OH}\right)$, and glycolaldehyde $\left(\mathrm{HOCH}_{2} \mathrm{CHO}\right)$ has been attributed to UV photolysis of condensed methanol found in interstellar ices. Experiments conducted in 1995 demonstrated that electron-induced radiolysis of methanol cosmic ice analogues yields methoxymethanol. In three recent publications (2016, 2017, and 2018), methoxymethanol was considered as a potential tracer for reactions induced by secondary electrons resulting from the interaction of cosmic rays with interstellar ices. However, the results presented in this study suggest that methoxymethanol can be formed from both radiation chemistry and photochemistry of condensed methanol.
\end{abstract}

Keywords: astrochemistry, radiation mechanisms: non-thermal, ISM: molecules, cosmic rays.

\subsection{Introduction}

Methoxymethanol $\left(\mathrm{CH}_{3} \mathrm{OCH}_{2} \mathrm{OH}\right)$ was

first detected in the interstellar medium in 2017 using the Atacama Large

Millimetre/submillimetre Array (ALMA) data in Bands 6 and 7 toward NGC 6334I, a massive protostar (McGuire et al. 2017). This finding is significant because the interstellar methoxymethanol abundance can help inform the relative yields of $\bullet \mathrm{CH}_{2} \mathrm{OH}$ and $\mathrm{CH}_{3} \mathrm{O}$ • radicals produced from methanol, which is thought to be an essential precursor to many prebiotic molecules (e.g., glycolaldehyde $\left(\mathrm{HOCH}_{2} \mathrm{CHO}\right)$ ) formed in interstellar ices (McGuire et al. 2017). Previous studies have identified methoxymethanol as an electroninduced radiolysis product of condensed methanol (Harris et al. 1995, Maity et al. 2015, Sullivan et al. 2016). Several previous studies did not report the identification of methoxymethanol as a photolysis product of methanol ices (Gerakines et al. 1996, Oberg et al. 2009, Allamandola 1988, Krim et al. 2009, Mrad et al. 2016). A tentative identification of methoxymethanol was claimed following irradiation of methanol cosmic ice analogs with vacuum UV photons capable of initiating both photochemistry and radiation chemistry (Paardekooper et al. 2016). In work reported herein, we provide persuasive evidence that photochemistry, in the absence of radiation chemistry, of condensed methanol yields methoxymethanol. Therefore, contrary to recent claims (McGuire et al. 2017, Motiyenko et al. 2018) based on previous claims (Boyer et al. 2016, Sullivan et al. 2016), methoxymethanol cannot be used as a tracer for electron-induced reactions initiated by cosmic rays in interstellar ices.

While some studies have concluded that most interstellar synthesis reactions take place in the gas phase (Charnley 1992), more

*Corresponding Author: Telephone: 781-283-3326; FAX: 781-283-3642; Email: carumain@wellesley.edu 
widely accepted research suggests that condensed-phase reactions must be included to match the observed abundances of interstellar prebiotic molecules (Tielens \& Hagen 1982, Garrod 2008). For some complex molecules such as formamide $\left(\mathrm{HCONH}_{2}\right)$, a gas-phase formation pathway has been suggested but is controversial (Skouteris et al. 2017, Song \& Kastner 2016). While non-energetic processing of ices, involving hydrogen atom accretion reactions, may lead to the synthesis of complex molecules such as glycerol

$\left(\mathrm{HOCH}_{2} \mathrm{CH}(\mathrm{OH}) \mathrm{CH}_{2} \mathrm{OH}\right)$, which can be formed through the hydrogenation of $\mathrm{CO}$ (Fedoseev et al. 2017), energetic processing of interstellar ices in dark, dense molecular clouds is thought to be the primary mechanism for the cosmic synthesis of prebiotic molecules. With temperatures as low as $10 \mathrm{~K}$, most gaseous molecules condense to form $\sim 100$ monolayerthick ices on micron-size dust grains. A likely route to prebiotic molecules involves energetic processing (photochemistry or radiation chemistry) of these cosmic ices. Although the external UV radiation field is unable to penetrate to the interior of dense molecular clouds (Prasad 1983), significant internal UV flux is observed in these dense clouds due to electronic relaxation of $\mathrm{H}_{2}$ following excitation by secondary electrons produced by cosmic rays (Gredel et al. 1989, Prasad 1983). In addition to being initiated by internal UV light, reactions may also be triggered by secondary electrons produced by the interation of cosmicrays with extraterrestrial ices. These nonthermal, low-energy $(<20 \mathrm{eV})$ electrons are considered to be the primary driving force in the radiolysis of cosmic ices (Boyer et al. 2016). Photochemistry/radiation chemistry of ice mantles yields heavy radicals such as - $\mathrm{CH}_{2} \mathrm{OH}$ and $\mathrm{CH}_{3} \mathrm{O}$ - that are thought to be immobile at $10 \mathrm{~K}$ because they cannot overcome the activation barrier for diffusion (Laas et al. 2011). During the warming process associated with the transition from a high/low mass prestellar core to a hot core/corino, complex organic molecules are synthesized through either (1) diffusion above $\sim 30 \mathrm{~K}$ of non-thermally formed radicals resulting in barrierless radical-radical reactions or (2) thermal reactions (e.g.,
$\mathrm{NH}_{3}+\mathrm{CO}_{2} \rightarrow \mathrm{NH}_{2} \mathrm{COOH}$ ) involving an activation barrier.

Because methanol abundances relative to water can be as high as 30\% in interstellar ices (Dartois et al. 1999), the photolysis products of methanol and their relative abundances are of interest to determine formation processes for more complex molecules. Reactions (1) and (2) shown below are two possible pathways for the electron/photon-induced dissociation of methanol.

$$
\begin{aligned}
& \mathrm{CH}_{3} \mathrm{OH} \rightarrow \cdot \mathrm{CH}_{2} \mathrm{OH}+\cdot \mathrm{H} \\
& \mathrm{CH}_{3} \mathrm{OH} \rightarrow \mathrm{CH}_{3} \mathrm{O} \cdot+\cdot \mathrm{H}
\end{aligned}
$$

Methoxymethanol $\left(\mathrm{CH}_{3} \mathrm{OCH}_{2} \mathrm{OH}\right)$ has been hypothesized to form via radical-radical reactions involving the hydroxymethyl radical $\left(\cdot \mathrm{CH}_{2} \mathrm{OH}\right)$ and the methoxy radical $\left(\mathrm{CH}_{3} \mathrm{O} \bullet\right)($ Harris et al. 1995, Laas et al. 2011, Boamah 2014):

$$
\mathrm{CH}_{3} \mathrm{O} \bullet+\cdot \mathrm{CH}_{2} \mathrm{OH} \rightarrow \mathrm{CH}_{3} \mathrm{OCH}_{2} \mathrm{OH}
$$

Due to the low abundances of these radicals in the gas phase restricting detection through rotational spectroscopy, the detection of methoxymethanol and its quantification can help determine the relative abundances of these radicals (McGuire et al. 2017). Chemical modeling of reaction 3 taking place in interstellar dust grains warming from $8-400 \mathrm{~K}$ over a period of $2.85 \times 10^{5}$ years gave a similar $\mathrm{CH}_{3} \mathrm{OCH}_{2} \mathrm{OH}: \mathrm{CH}_{3} \mathrm{OH}$ ratio as the observed ratio of 1:34 (McGuire et al. 2017).

Several studies have used microwave discharge hydrogen flow lamps (MDHL) (dominated by the Lyman- $\alpha$ peak at $10.2 \mathrm{eV}$ and the Lyman band system of molecular hydrogen with peaks at 7.7 and $7.9 \mathrm{eV}$ ) to mimic condensed-phase methanol UV chemistry in the interstellar medium (Gerakines et al. 1996, Oberg et al. 2009, Allamandola et al.1988, Paardekooper et al. 2016). In another UV study, the photon source used was a krypton lamp whose emission spectrum is composed of two intense peaks at $10.6 \mathrm{eV}$ and $10.0 \mathrm{eV}$ and a broad continuum in the 7.3-9.5 eV range (Krim et al. 2009). Together, one or more of the above mentioned studies involving UV and VUV photon irradiation of condensed 
methanol have demonstrated the formation of acetaldehyde $\left(\mathrm{CH}_{3} \mathrm{CHO}\right)$, glycolaldehyde $\left(\mathrm{HOCH}_{2} \mathrm{CHO}\right)$, methyl formate $\left(\mathrm{HCOOCH}_{3}\right)$, formic acid $(\mathrm{HCOOH})$, acetic acid $\left(\mathrm{CH}_{3} \mathrm{COOH}\right)$, ethylene glycol $\left(\left(\mathrm{CH}_{2} \mathrm{OH}\right)_{2}\right)$, dimethyl ether $\left(\mathrm{CH}_{3} \mathrm{OCH}_{3}\right)$, ethanol $\left(\mathrm{CH}_{3} \mathrm{CH}_{2} \mathrm{OH}\right)$, formaldehyde $\left(\mathrm{H}_{2} \mathrm{CO}\right)$, carbon monoxide $(\mathrm{CO})$, methane $\left(\mathrm{CH}_{4}\right)$, and carbon dioxide $\left(\mathrm{CO}_{2}\right)$. To the best of our knowledge, only one study reported the tentative identification of methoxymethanol as a product of methanol ices subjected to VUV photoprocessing which likely involves both photochemistry and radiation chemistry (Paardekooper et al. 2016).

Photochemical reactions, by definition, are initiated by molecules in excited electronic states (Wardle 2009). Molecules typically reach excited states through absorption of visible, near-UV, and far-UV photons (Wardle 2009). Such photons have energies between 1.8 and $6.2 \mathrm{eV}$, below the ionization threshold of most molecules. Vacuum-UV $(6.2-12.4 \mathrm{eV})$ light may initiate, in addition to photochemistry, radiation chemistry, which, unlike photochemistry, involves higher energy, ionizing radiation. The incident photon energy must be below the ionization threshold of the substance to perform photochemistry studies in the absence of radiation chemistry. Gas-phase methanol has an ionization energy of $10.8 \mathrm{eV}$ (Lias 2005), which is reduced by $\sim 1.0 \mathrm{eV}$ in the condensed phase (Yu et al. 1975), leading to a condensed-phase ionization threshold energy of $\sim 9.8 \mathrm{eV}$.

Here, by using a photon source which produces photons with energies $<7.4 \mathrm{eV}$, we identify methoxymethanol as a pure photochemistry product of condensed methanol. Specifically, we demonstrate that methoxymethanol can form from methanol ices by photon irradiation without the involvement of secondary electrons and/or low-energy cations produced by ionization of condensed methanol. Therefore, methoxymethanol cannot be used as a tracer molecule for electroninitiated radiation chemistry reactions in interstellar ices.

\subsection{Experimental}

Experiments were performed in a stainless steel ultrahigh vacuum (UHV) chamber with a base pressure of $2 \times 10^{-9}$ Torr, previously described in detail (Harris et al. 1995). A Ta(110) crystal mounted on a rotary manipulator was used as the substrate for experiments. The crystal was cleaned prior to each experiment by electron bombardment heating to $\sim 2200 \mathrm{~K}$ for $\sim 30$ seconds.

Liquid nitrogen was used to cool the crystal to approximately $90 \mathrm{~K}$ before dosing methanol on the crystal. Samples were obtained from Sigma-Aldrich (methanol, HPLC grade $99.9 \%$ ). Liquid samples were transferred to Schlenk tubes and degassed by three freezepump-thaw cycles.

The methanol films were irradiated using a laser-driven low-energy photon source described previously (Mullikin et al. 2018). The source has an upper limit of $7.4 \mathrm{eV}$ (Kuo et al. 2007) and the lower limit of the absorption spectrum of condensed methanol is $6.7 \mathrm{eV}$. We conclude that only photons with energies 6.7$7.4 \mathrm{eV}$ are likely effective in the formation of the detected methanol photolysis products.

After irradiation, photolysis products were separated based on desorption temperatures using temperature-programmed desorption experiments conducted by heating the crystal to $800 \mathrm{~K}$. Products were monitored using a triple-filtered Hiden Ion Desorption Probe Series 500 quadrupole mass spectrometer. Post-irradiation temperatureprogrammed desorption experiments were conducted with methanol isotopologues to further verify peak assignments.

Photochemistry was confirmed to be the only process involved in product formation (Mullikin et al. 2018). The maximum kinetic energy of a photoejected electron from the crystal surface is below the minimum electron energy $(\sim 5 \mathrm{eV})$ required to form methoxymethanol from methanol (Boyer et al. 2014), indicating that the observed formation of methoxymethanol and ethylene glycol is due only to the incident photons.

\subsection{Results and Discussion}

We present novel data identifying methoxymethanol $\left(\mathrm{CH}_{3} \mathrm{OCH}_{2} \mathrm{OH}\right)$ as a product of condensed methanol irradiated with photons 
of energies $<7.4 \mathrm{eV}$, below the ionization threshold of condensed methanol. As a control, temperature-programmed desorption experiments were conducted with unirradiated methanol films. The peak at $\sim 140 \mathrm{~K}$ (Fig. 1) is attributed to methanol desorption; due to the natural $\sim 1 \%$ abundance of ${ }^{13} \mathrm{C}$, this peak is observed for $m / z 33$. At the same temperature, the peaks observed for $m / z 45,61$ and 62 are attributed to ion-molecule gas-phase reactions of methanol within the ionizer of the mass spectrometer. Methanol reactions on the $\mathrm{Ta}(110)$ surface do not yield products that have any of the monitored mass spectral fragments (Fig. 1).

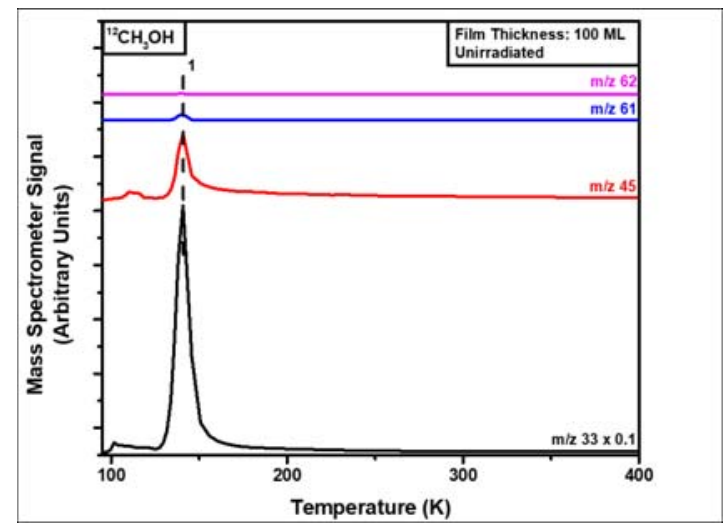

Figure 1: Temperature-programmed desorption experiment performed on an unirradiated sample of 100 monolayers of ${ }^{12} \mathrm{CH}_{3} \mathrm{OH}$. The peak at $\sim 140 \mathrm{~K}$ (1) is attributed to methanol desorption. The emission current of the mass spectrometer was set to $300 \mu \mathrm{A}$ for $m / z 33$, and $1300 \mu \mathrm{A}$ for all other mass to charge ratios. Plots vertically offset for clarity.

After irradiation with low-energy $(\leq$ $7.4 \mathrm{eV}$ ) photons with a total fluence of $7.2 \times$ $10^{18} \mathrm{~cm}^{-2}$, methanol photolysis products were identified using temperature-programmed desorption (Fig. 2). The identification of ethylene glycol $\left(\mathrm{HOCH}_{2} \mathrm{CH}_{2} \mathrm{OH}\right)$ was based on peaks for $m / z=33\left[\mathrm{CH}_{5} \mathrm{O}^{+}\right], m / z=45\left[\mathrm{C}_{2} \mathrm{H}_{4} \mathrm{O}^{+}\right]$ and $\mathrm{m} / \mathrm{z}=61\left[\mathrm{OCH}_{2} \mathrm{CH}_{2} \mathrm{OH}^{+}\right]$at $\sim 205 \mathrm{~K}$ observed in the results of post-irradiation temperature-programmed desorption experiments (Fig. 2). These three mass to charge ratios are significant fragments in the mass spectrum of ethylene glycol (NIST Mass Spec Data Center \& Stein). The identification of ethylene glycol was further confirmed by the results of temperature-programmed desorption experiments involving an unirradiated film of a mixture of $\sim 10 \%(\mathrm{v} / \mathrm{v})$ ethylene glycol in ${ }^{12} \mathrm{CH}_{3} \mathrm{OH}$ (Supplementary Information, Fig. $\mathrm{S} 1)$. The mass spectral peaks observed in these experiments were consistent with those attributed to methanol and ethylene glycol in the experiments involving irradiation. The first peak at $\sim 100 \mathrm{~K}$ with a major fragment at $\mathrm{m} / \mathrm{z}$ 45 is attributed to dimethyl ether based on previous studies (Harris et al. 1995, Boamah 2014).

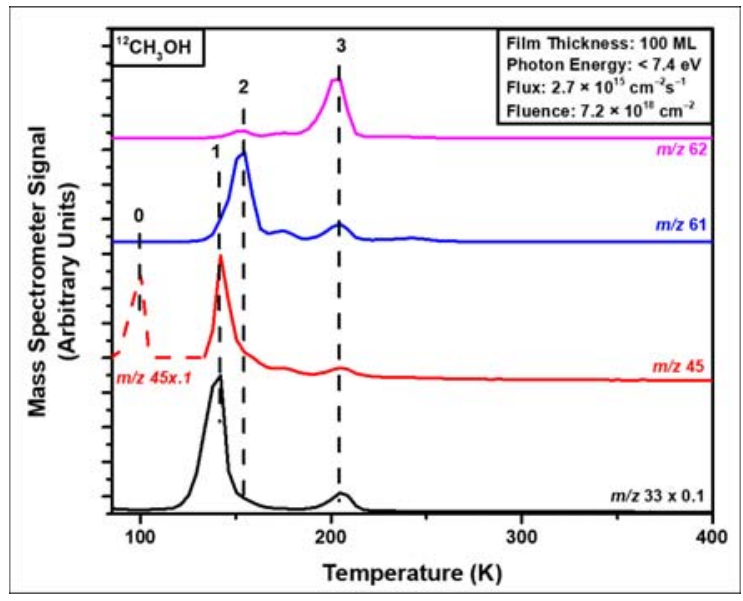

Figure 2: Post-irradiation temperature-programmed desorption data for 100 monolayers of ${ }^{12} \mathrm{CH}_{3} \mathrm{OH}$ irradiated with low-energy UV photons with a total fluence of $7.2 \times 10^{18} \mathrm{~cm}^{-2}$. The peak at $\sim 100 \mathrm{~K}(0)$ is attributed to dimethyl ether $\left(\mathrm{CH}_{3} \mathrm{OCH}_{3}\right)(\mathrm{m} / \mathrm{z}=45)$, the peak at $\sim 140 \mathrm{~K}(1)$ is attributed to methanol $(\mathrm{m} / \mathrm{z}$ $=33,45)$, the peak at $\sim 155 \mathrm{~K}(2)$ is attributed to methoxymethanol $\left(\mathrm{CH}_{3} \mathrm{OCH}_{2} \mathrm{OH}\right)(\mathrm{m} / \mathrm{z}=61$ and 62$)$, and the peak at $205 \mathrm{~K}(3)$ is attributed to ethylene glycol $\left(\left(\mathrm{CH}_{2} \mathrm{OH}\right)_{2}\right)(\mathrm{m} / \mathrm{z}=33,45,61,62)$. The emission current of the mass spectrometer was set to $300 \mu \mathrm{A}$ for $\mathrm{m} / \mathrm{z} 33$, and $1300 \mu \mathrm{A}$ for all other mass to charge ratios. Plots vertically offset for clarity.

\subsection{Identification of Methoxymethanol}

The post-irradiation desorption features at $\sim 155 \mathrm{~K}$ for $\mathrm{m} / \mathrm{z}=61\left[{ }^{12} \mathrm{C}_{2} \mathrm{H}_{5} \mathrm{O}_{2}{ }^{+}\right]$and 62 $\left[{ }^{12} \mathrm{C}_{2} \mathrm{H}_{6} \mathrm{O}_{2}^{+}\right]$are assigned to methoxymethanol (Fig. 2). The observed relative peak intensity for $m / z=61$ and 62 are consistent with the published methoxymethanol mass spectrum which shows that the 61 fragment intensity is significantly higher than that of 62 (Johnson \& Stanley 1991). Peak shoulders for $m / z=31$ and 45 , two important mass spectral fragments of methoxymethanol, also align with the desorption features for methoxymethanol. Our identification of methoxymethanol as a 
photochemistry product of methanol is further corroborated by methanol radiation chemistry experiments done in the same UHV chamber. In experiments conducted following electron irradiation of methanol ices, desorption features at $\sim 155 \mathrm{~K}$ were observed for $\mathrm{m} / \mathrm{z}=$ $61\left[{ }^{12} \mathrm{C}_{2} \mathrm{H}_{5} \mathrm{O}_{2}^{+}\right]$and $62\left[{ }^{12} \mathrm{C}_{2} \mathrm{H}_{6} \mathrm{O}_{2}^{+}\right]$with approximately the same intensity ratio as seen following photochemistry. Electron-induced methanol radiolysis to yield methoxymethanol has been previously confirmed via multiple techniques in two independent laboratories (Harris et al. 1995 and Maity et al. 2015). Additionally, in experiments conducted with ${ }^{13} \mathrm{CH}_{3} \mathrm{OH}$ (Fig. 3), following irradiation with a total photon fluence of $5.3 \times 10^{18} \mathrm{~cm}^{-2}$, peaks for $m / z=63\left[{ }^{13} \mathrm{C}_{2} \mathrm{H}_{5} \mathrm{O}_{2}{ }^{+}\right], m / z=64\left[{ }^{13} \mathrm{C}_{2} \mathrm{H}_{6} \mathrm{O}_{2}{ }^{+}\right]$, and $\mathrm{m} / \mathrm{z}=34\left[{ }^{13} \mathrm{CH}_{3} \mathrm{OH}_{2}{ }^{+}\right]$were observed at $\sim 155 \mathrm{~K}$. The relatively high $\mathrm{m} / \mathrm{z}=63$ intensity at $\sim 155 \mathrm{~K}$ is likely not explained by the desorption of another species (e.g., acetic acid; protonated $\mathrm{C}-13$ acetic acid has an $\mathrm{m} / \mathrm{z}=63$ fragment) because of the near-perfect match between the $m / z=63$ and $m / z=64$ desorption profiles. While the discrepancy between $\mathrm{m} / \mathrm{z}=$ 63:64 and $m / z=61: 62$ signals at $\sim 155 \mathrm{~K}$ maybe explained by differences in fragmentation intensities, quadrupole transmission factors, and detector efficiencies, we cannot unequivocally rule out the possibility that an unidentified photolysis product desorbs at $155 \mathrm{~K}$. Methoxymethanol identification was further confirmed by $\mathrm{CD}_{3} \mathrm{OD}$ post-irradiation temperature programmed desorption experiments which evinced desorption peaks at $\sim 155 \mathrm{~K}$ for $\mathrm{m} / \mathrm{z}=66\left[{ }^{12} \mathrm{C}_{2} \mathrm{D}_{5} \mathrm{O}_{2}{ }^{+}\right]$and $\mathrm{m} / \mathrm{z}=68$ $\left[{ }^{12} \mathrm{C}_{2} \mathrm{D}_{6} \mathrm{O}_{2}{ }^{+}\right]$. A recently reported tentative identification of methoxymethanol involved methanol ices subject to VUV processing which likely involves both photochemistry and radiation chemistry (Paardekooper et al. 2016). According to this publication, "In the 150-175 $\mathrm{K}$ temperature window, we do find a decrease in the dominant peaks consistent with methoxymethanol, indicating that it may be formed."

While the interiors of dark, dense molecular clouds reach temperatures as low as $10 \mathrm{~K}$, they warm to $>100 \mathrm{~K}$ during the transition from the cold phase to a hot core or corino over a period of a million years.
Temperatures exceeding $\sim 30 \mathrm{~K}$ are sufficient for barrierless radical-radical reactions of nonthermally formed radicals such as $\bullet \mathrm{CH}_{2} \mathrm{OH}$ and $\mathrm{CH}_{3} \mathrm{O} \cdot$. Therefore, our studies have astrochemical implications even though we use a high sample temperature of $90 \mathrm{~K}$. In addition, our results demonstrate that methoxymethanol formation is not restricted to radiation chemistry which may involve ion-molecule reactions such as $\mathrm{CH}_{3} \mathrm{OH}+\left[\mathrm{CH}_{3} \mathrm{OH}\right]^{+} \rightarrow$ - $\mathrm{CH}_{2} \mathrm{OH}+\left[\mathrm{CH}_{3} \mathrm{OH}_{2}\right]^{+}$. Rather, our results demonstrate that electronic excitation of methanol likely yields $\bullet \mathrm{CH}_{2} \mathrm{OH}$ and $\mathrm{CH}_{3} \mathrm{O} \bullet$, whose subsequent recombination yields methoxymethanol.

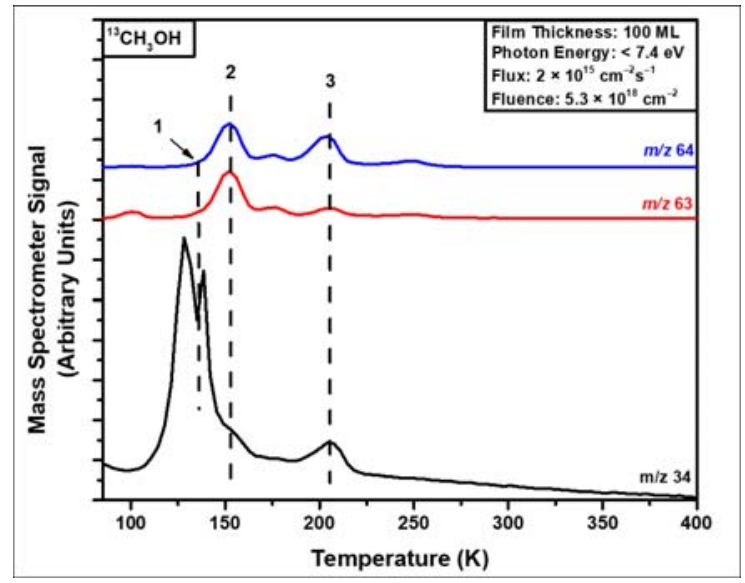

Figure 3: Post-irradiation temperature-programmed desorption data for 120 monolayers of ${ }^{13} \mathrm{CH}_{3} \mathrm{OH}$ irradiated with a total photon fluence of $5.3 \times 10^{18}$ $\mathrm{cm}^{-2}$. The peak at $\sim 140 \mathrm{~K}(1)$ is attributed to methanol $(\mathrm{m} / \mathrm{z}=34)$, the peak at $\sim 155 \mathrm{~K}(2)$ is attributed to methoxymethanol $\left(\mathrm{CH}_{3} \mathrm{OCH}_{2} \mathrm{OH}\right)(\mathrm{m} / \mathrm{z}=$ 34,63 and 64), and the peak at $205 \mathrm{~K}$ (3) is attributed to ethylene glycol $\left(\left(\mathrm{CH}_{2} \mathrm{OH}\right)_{2}\right)(\mathrm{m} / \mathrm{z}=34$, $63,64)$. The dip at $\sim 140 \mathrm{~K}$ is due to detector saturation. Plots vertically offset for clarity.

\subsection{Conclusions}

Results of our post-irradiation temperatureprogrammed desorption experiments demonstrate that photochemistry of condensed methanol leads to the formation of methoxymethanol whose first detection in the interstellar medium was reported in 2017. The use of a $\leq 7.4 \mathrm{eV}$ photon source in our experiments ensured that low-energy secondary electrons and/or methanol cations did not play a role in the synthesis of the observed methoxymethanol. Rather, we suggest that electronic excitation followed by dissociation 
of methanol yields the hydroxymethyl radical $\left(\cdot \mathrm{CH}_{2} \mathrm{OH}\right)$ and the methoxy radical $\left(\mathrm{CH}_{3} \mathrm{O} \cdot\right)$ whose reaction produces methoxymethanol. Because methoxymethanol can be formed from both the radiation chemistry and

photochemistry of methanol, methoxymethanol cannot be an indicator of electron-induced ice chemistry in the interstellar medium.

\section{Acknowledgments}

This work was supported by grants from the National Science Foundation (NSF grant number CHE-1465161), Wellesley College (Faculty awards and Brachman Hoffman small grants), and Clark University (Sherman Fairchild Summer Scholars Program and the Physics Department).

Supplementary material is available online.

\section{References}

Abou Mrad N., Duvernay, F., Chiavassa, T., Danger, G., 2016, Mon Not R Astron Soc, 458, 1234.

Allamandola L.J., Sandford, S. A., Valero, G. J., 1988, Icarus, 76, 225

Boamah M.D., Sullivan, K. K., Shulenberger, K. E., Soe, C. M., Jacobs, L. M., Yhee, F. C., Atkinson, K. E., Boyer, M. C., Haines, D. R., Arumainayagam, C. R., 2014, Faraday Discuss, 168

Boyer M.C., Boamah M.D., Sullivan K.K., Arumainayagam C.R., Bazin M., Bass A.D., Sanche L., 2014, The Journal of Physical Chemistry C, 118, 22592

Boyer M.C., Rivas N., Tran A.A., Verish C.A., Arumainayagam C.R., 2016, Surface Science, 652, 26

Charnley S.B., Tielens, A.G.G.M., Millar, T.J. , 1992, ApJ, 399, L71

Christian George M.A., Barbara D'Anna, D. J. Donaldson, Sergey A. Niskorodov, 2015, Chem. Rev., 115, 4218

Dartois E., Schutte W., Geballe T.R., Demyk K., Ehrenfreund P., d'Hendecourt L., 1999, Astronomy \& Astrophysics, 342, L32

Derry G.N., Kern M.E., Worth E.H., 2015, Journal of Vacuum Science \&
Technology A: Vacuum, Surfaces, and Films, 33, 060801

Fedoseev G., Chuang K., loppolo S., Qasim D., van Dishoeck E., Linnartz H., 2017, Astrophysical Journal, 842

Garrod R.T., Widicus, S.L., Herbst, E., 2008, ApJ, 682, 283

Gerakines P., Schutte W., Ehrenfreund P., 1996, Astronomy \& Astrophysics, 312, 289

Gredel R., Lepp S., Dalgarno A., Herbst E., 1989, Astrophysical Journal, 347, 289

Harris T.D., Lee D.H., Blumberg M.Q., Arumainayagam C.R., 1995, J. Phys. Chem., 99, 9530

Johnson R.A., Stanley A.E., 1991, Appl. Spectrosc., 45, 218

Krim L., Lasne J., Laffon C., Parent P., 2009, Journal of Physical Chemistry a, 113, 8979

Kuo Y., Lu H., Wu Y., Cheng B., Ogilvie J., 2007, Chemical Physics Letters, 447, 168

Laas J.C., Garrod R.T., Herbst E., Weaver S.L.W., 2011, Astrophysical Journal, 728,1

Lias S.G., in: P. J. Linstrom W.G.M. ed., NIST Standard Reference Database Number 69, 2005, National Institute of Standards and Technology, Gaithersburg MD, 20899,

Maity S., Kaiser R.I., Jones B.M., 2015, Physical Chemistry Chemical Physics, 17, 3081

McGuire B., et al., 2017, Astrophysical Journal Letters, 851

Mullikin E., et al., 2018, ACS Earth and Space Chemistry, 2, 9

NIST Mass Spec Data Center, Stein S.E., in: Linstrom P.J., Mallard W.G. eds., NIST Chemistry WebBook, NIST Standard Reference Database Number 69. National Institute of Standards and Technology, Gaithersburg MD,

Oberg K., Garrod R., van Dishoeck E., Linnartz H., 2009, Astronomy \& Astrophysics, 504,891

Paardekooper D.M., Bossa J.-B., Linnartz, H., 2016, Astronomy \& Astrophysics, 592, A67 
Prasad S.S., Tarafdar, S.P., 1983, Astrophysical Journal, 267, 603

Skouteris D., Vazart F., Ceccarelli C., Balucani N., Puzzarini C., Barone V., 2017, Monthly Notices of the Royal Astronomical Society: Letters, 468, L1

Song L., Kastner J., 2016, Physical Chemistry Chemical Physics, 18, 29278

Sullivan K.K., Boamah M.D., Shulenberger K.E., Chapman S., Atkinson K.E., Boyer M.C., Arumainayagam C.R., 2016, Mon Not R Astron Soc, 460, 664

Tielens A. G. G. M., Hagen W., 1982, Astronomy and Astrophysics, 114

Wardle B., 2009, John Wiley \& Sons, Incorporated, New York, UNITED KINGDOM

YU K., MCMENAMIN J., SPICER W., 1975, Surface Science, 50, 149 